\title{
Descarte das bolsas produzidas em um centro de hemoterapia de uma região do Nordeste do Brasil
}

\author{
Disposal of bags produced in a hemotherapy center in a Northeastern region of Brazil \\ Eliminación de bolsas producidas en un centro de hemoterapia en una región Noreste de Brasil
}

Recebido: 11/08/2021 | Revisado: 15/08/2021 | Aceito: 16/08/2021 | Publicado: 18/08/2021

Ana Iris Mykaella Santos Sousa

ORCID: https://orcid.org/0000-0001-8347-0603

Universidade Tiradentes, Brasil

E-mail: anairismss@outlook.com

Alexia Rachel Portugal Lemos Oliveira

ORCID: https://orcid.org/0000-0001-9587-8518

Universidade Tiradentes, Brasil

E-mail: alexiarplo@outlook.com

Weber de Santana Teles

ORCID: https://orcid.org/0000-0003-1770-8278

Centro de Hemoterapia de Sergipe, Brasil

E-mail: arteecura@hotmail.com

Max Cruz da Silva

ORCID: https://orcid.org/0000-0002-6944-5986

Faculdade Pio Décimo, Brasil

E-mail: maxlfi@hotmail.com

Paulo Celso Curvelo Santos Junior

ORCID: https://orcid.org/0000-0001-5834-6782

Universidade Tiradentes, Brasil

E-mail: paulo.curvelo.jr@gmail.com

Ruth Cristini Torres

ORCID: https://orcid.org/0000-0002-8664-192X

Instituto de Hematologia e Hemoterapia de Sergipe, Brasil

E-mail: ruthcristini@gmail.com

Maria Hozana Santos Silva

ORCID: https://orcid.org/0000-0001-5742-5366

Faculdade Ages de Medicina, Brasil

E-mail: hosana_p@hotmail.com

Marcel Vinícius Cunha Azevedo

ORCID: https://orcid.org/0000-0002-5312-3333

Centro Universitário Estácio Sergipe, Brasil

E-mail: marcelvinicius49@gmail.com

Ângela Maria Melo Sá Barros

ORCID: https://orcid.org/0000-0003-4087-3247

Universidade Federal do Rio de Janeiro, Brasil

E-mail: angelsamelo@hotmail.com

Ana Fátima Souza Melo de Andrade

ORCID: https://orcid.org/0000-0002-7024-6175

Centro Universitário Estácio de Sergipe, Brasil

E-mail: anafatimamelo@hotmail.com

Alejandra Debbo

ORCID: https://orcid.org/0000-0002-7743-5921

Universidade Tiradentes, Brasil

E-mail: aledebbo@hotmail.com

Taíssa Alice Soledade Calasans

ORCID: https://orcid.org/0000-0003-0460-4437

Universidade Tiradentes, Brasil

E-mail: taissa.asc@gmail.com

\section{Resumo}

O presente trabalho teve como objetivos analisar a frequência do descarte das bolsas produzidas em um centro de hemoterapia de uma região do nordeste do Brasil. Trata-se de uma análise retrospectiva de candidatos à doação de sangue, no período no período de setembro de 2107 a agosto de 2018. Os dados foram extraídos do software do setor de informática do Hemocentro e organizados e classificados no programa Excel 365 E pelo software IBM Statistical 
Package for the Social Sciences, 22.0. No período compreendido entre de setembro de 2017 a agosto de 2018, foram realizadas 28.800 destas, $5.8289(20,2 \%)$, sendo $21,1 \%$ (1.229) foram referentes ao descarte de concentrados de hemácias, 41,5\% (2419), concentrado de plaquetas, 36,2\% (2.111) concentrado de plasma e o sangue total 1,2\% (69). Os motivos dos expurgos foram em recorrência de variáveis como intercorrência no fracionamento, intercorrência na distribuição, expiração do prazo de validade, acondicionamento inadequado, sorologia reagente, coombs indireto e intercorrência no estoque. Os resultados do presente estudo propõem um plano de ação, envolvendo a reavaliação dos protocolos operacionais, com avaliações periódicas das demandas de descarte, visando readequar a oferta e reduzir ou até extinguir o descarte de bolsas de hemocomponentes, o que direcionaria a uma redução de custos financeiros e perdas sociais.

Palavras-chave: Bancos de sangue; Bolsas plásticas para preservação de sangue; Serviço de hemoterapia.

\begin{abstract}
This study aimed to analyze the frequency of disposal of bags produced in a hemotherapy center in a region of northeastern Brazil. This is a retrospective analysis of candidates for blood donation, in the period from September 2107 to August 2018. Data were extracted from the software of the information technology sector of the Blood Center and organized and classified in the Excel 365 E program by the software IBM Statistical Package for the Social Sciences, 22.0. In the period from September 2017 to August 2018, 28,800 of these were carried out, 5,8289 (20.2\%), of which $21.1 \%(1,229)$ were related to the disposal of red blood cell concentrates, $41.5 \%$ (2419), platelet concentrate, $36.2 \%$ (2,111) plasma concentrate and whole blood $1.2 \%$ (69). The reasons for the purges were due to variables such as complications in fractionation, problems in distribution, expiration of the shelf life, inadequate packaging, reagent serology, indirect coombs and problems in the stock. The results of this study propose an action plan, involving the reassessment of operational protocols, with periodic assessments of disposal demands, aiming to readjust the supply and reduce or even extinguish the disposal of blood component bags, which would lead to a reduction in costs financial and social losses.
\end{abstract}

Keywords: Blood banks; Plastic bags for blood preservation; Hemotherapy service.

\title{
Resumen
}

Este estudio tuvo como objetivo analizar la frecuencia de eliminación de bolsas producidas en un centro de hemoterapia en una región del noreste de Brasil. Se trata de un análisis retrospectivo de candidatos a donación de sangre, en el período de septiembre de 2107 a agosto de 2018. Los datos fueron extraídos del software del sector de tecnologías de la información del Centro de Sangre y organizados y clasificados en el programa Excel 365 E por el software IBM Paquete estadístico para las ciencias sociales, 22.0. En el período de septiembre de 2017 a agosto de 2018 se realizaron 28.800 de estos, 5.8289 (20,2\%), de los cuales el 21,1\% (1.229) correspondieron a la disposición de concentrados de hematíes, 41,5\% (2419), plaquetas concentrado, 36,2\% (2,111) concentrado de plasma y sangre total 1,2\% (69). Los motivos de las purgas se debieron a variables como complicaciones en el fraccionamiento, problemas en la distribución, fecha de vencimiento, empaque inadecuado, serología de los reactivos, coombs indirectos y problemas de stock. Los resultados de este estudio proponen un plan de acción, que implica la reevaluación de los protocolos operativos, con evaluaciones periódicas de las demandas de eliminación, con el objetivo de reajustar el suministro y reducir o incluso extinguir la eliminación de bolsas de componentes sanguíneos, lo que conllevaría una reducción de los costos financieros. y pérdidas sociales.

Palabras clave: Bancos de sangre; Bolsas plásticas para conservación de sangre; Servicio de hemoterapia.

\section{Introdução}

Os produtos concebidos nos procedimentos de hemoterapia, a partir do sangue total por meio do centrifugador são alcunhados hemocomponentes, e o fracionamento do sangue total são designados de hemoderivados. Ambos os produtos são usufruídos tencionando assistir indivíduos em mediação cirúrgicas e disfunções da coagulação sanguínea, e para que a seguridade do doador e do receptor sejam assegurados, postula que as fases referentes ao ciclo do sangue sejam atendidas de acordo com as normas e legislações vigentes (Who, 2017).

A Secretária de Estado da Saúde de Porto Alegre (2020), através plano estadual de saúde, informa que a Agência Nacional de Vigilância Sanitária (ANVISA), através da Gerência de Sangue, Tecidos, Células e Órgãos - GSTCO, fiscaliza e vistoria os procedimentos operacionais que tem relação com o sangue, estabelecendo legislações para as unidades de hemoterapia que integram a hemorrede nacional, constituída por hemocentro coordenador, regionais, hemonúcleos e unidades de coleta e transfusão. 
A procedimentos operacionais padrão preconizados pelos regulamentos sanitários no que diz respeito ao cadastro, prétriagem laboratorial e clínica, coleta, produção do sangue, e exames de fenotipagem eritrocitária de doadores e receptores de sangue e a avaliação sorológicas devem ser atendidos para que aja uma logística de qualidade dos serviços prestados, assim como a implantação e implementação de comitê técnico para acompanhamento, vigilância e controle (Brasil, 2016).

A triagem clínica tem uma função vultuosa na avaliação do candidato a doação de sangue, a aferição da pressão arterial, dosagem de hemoglobina e a entrevista que consiste em uma anamnese do padrão comportamental entre outros, são passos que carecem de ser atendidos para que o candidato a doação possa seguir para o setor de coleta de sangue (Dias, et al., 2019).

Segundo os dados da totalidade de doações sanguíneas, no Brasil no ano de 2017, foram realizadas 3,4 milhões de coletas de sangue e foram transfundidos cerca de 2,8 milhões de bolsas de sangue (Ventura, 2019).

A Organização Pan-Americana da Saúde (OPAS) (2017), demonstra que os descartes das bolsas de sangue devido às não conformidades encontradas pelos protocolos dos serviços de controle de qualidade levam às notáveis perdas financeiras e sociais, o que leva a um aumento dos custos na manutenção dos sistemas de coletas e produção de hemoderivados e hemocomponentes. As perdas financeiras são relativamente claras e dimensíveis, qualificadas pelas horas de trabalho gastas pelos funcionários, uso de equipamentos, energia elétrica, estrutura física entre outros recursos destinados à coleta e processamento de sangue e hemoderivados.

Os prejuízos sociáveis são definidos pelo investimento que se perdeu em esfera física, emocional e do tempo gasto pelas pessoas que se dispõem a realizar doações, e estas são perdas imensuráveis e intangíveis (Covo, et al., 2019). Como proposta para redução do descarte, encontrada na literatura, faz-se necessárias campanhas educativas relatando a inaptidão sorológica, bem como, propor melhores formas na gestão de validade do estoque, facilitando a identificação e diferenciação dos hemocomponentes, reduzindo as taxas de descarte (Palludeto et al., 2015).

Baseado nisto, o presente trabalho possui como objetivos analisar o descarte de bolsas de sangue e compreender as principais causas da inaptidão dessas bolsas coletadas, armazenadas e distribuídas pelo Centro de Hemoterapia de Sergipe, no período compreendido entre o período de setembro de 2017 a agosto de 2018.

\section{Metodologia}

Trata-se de uma análise retrospectiva de dados de 5.828 bolsas de candidatos à doação de sangue obtidas no setor de coleta e processadas no setor de produção e dispensação de hemocomponentes, no período de setembro de 2107 a agosto de 2018. Os dados foram extraídos do software do setor de informática do Hemocentro e organizados e classificados no programa Excel 365. Após a organização, a análise estatística deu-se pelo software IBM Statistical Package for the Social Sciences, 22.0, utilizando um intervalo de confiança de 95\% ( $\mathrm{p}<0.05)$ para significância estatística, e teste Kruskal-Wallis de amostras independentes.

\section{Resultados}

No período compreendido entre de setembro de 2017 a agosto de 2018, foram realizadas 28.800 destas, 5.8289 (20,2\%), sendo 21,1\% (1.229) foram referentes ao descarte de concentrados de hemácias, 41,5\% (2419), concentrado de plaquetas, 36,2\% (2.111) concentrado de plasma e o sangue total 1,2\% (69). (Figura 1). 
Figura 1: Prevalência do descarte de concentrados de hemácias, plaquetas, plasma e sangue total durante o período de setembro de 2017 a agosto de 2018 no Centro de Hemoterapia de Sergipe.

\section{DESCARTE DOS CONCENTRADOS}

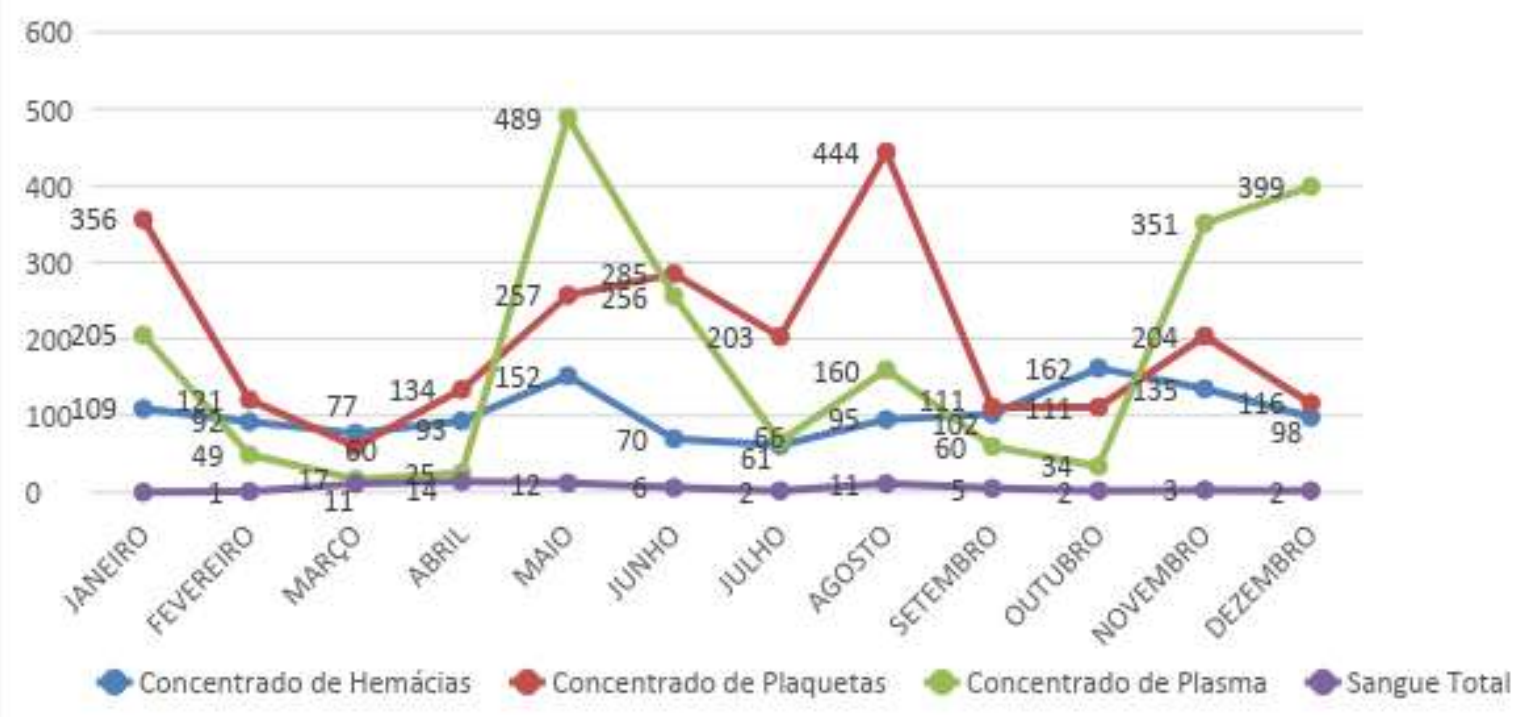

Fonte: Autores.

Em relação ao quantitativo de descarte dos concentrados, notou-se um valor médio de 16 concentrados de hemácias descartados, enquanto, o maior valor médio de descarte, foi relacionado aos concentrados de plaquetas. Ao verificar o valor máximo descartado, notou-se que o maior índice de descarte responsável foi relacionado ao concentrado de plasma, notando-se uma significância estatística na distribuição dos concentrados em relação ao descarte total (p<0,05) (Quadro 1).

Quadro 1: Quantidade média de descarte dos concentrados durante o período de setembro de 2017 a agosto de 2018 no Centro de Hemoterapia de Sergipe.

\begin{tabular}{|r|c|c|c|c|}
\hline \multirow{2}{*}{ TIPOS } & \multicolumn{2}{|c|}{ TERMOS DE TENDÊNCIA CENTRAL } & \multirow{2}{*}{$\mathrm{p}^{*}$} \\
\cline { 2 - 4 } & MEDIANA & MÍNIMO & MÁXIMO & \\
\hline CH & 16 & 1 & 143 & \\
\hline CP & 111 & 1 & 444 & \\
\hline CPlasma & 63 & 1 & 14 & \multirow{2}{*}{$\mathbf{0 . 0 1 5}$} \\
\hline ST & 5 & 1 & & \\
\hline
\end{tabular}

* Kruskal-Wallis de Amostras Independentes. * CH: concentrado de hemácias; CP: concentrado de plaquetas; CPlasma: Concentrado de plasma; ST: sangue total.

Fonte: Autores.

Quando analisados em relação ao acondicionamento inadequado e prazo de validade expirado foi observado maior índice de inadequação no concentrado de plasma, seguido de concentrado de plaquetas (Quadro 2). 
Quadro 2: Descartes dos concentrados devido o acondicionamento inadequado e prazo de validade expirada durante o período de setembro de 2017 a agosto de 2018 no Centro de Hemoterapia de Sergipe.

\begin{tabular}{|c|c|c|c|c|}
\hline \multicolumn{5}{|c|}{ ACONDICIONAMENTO INADEQUADO } \\
\hline TIPOS & MEDIANA & MÍNIMO & MÁXIMO & \multirow{5}{*}{$\mathbf{0 . 0 0 3}$} \\
\hline $\mathrm{CH}$ & - & - & - & \\
\hline $\mathrm{CP}$ & - & - & - & \\
\hline CPlasma & 1 & 0 & 8 & \\
\hline ST & - & - & - & \\
\hline \multicolumn{4}{|c|}{ PRAZO DE VALIDADE EXPIRADA } & \multirow{6}{*}{$<0.001$} \\
\hline TIPOS & MEDIANA & MÍNIMO & MÁXIMO & \\
\hline $\mathrm{CH}$ & 1 & 0 & 32 & \\
\hline $\mathrm{CP}$ & 24 & 1 & 299 & \\
\hline CPlasma & 1 & 0 & 1 & \\
\hline ST & - & - & - & \\
\hline
\end{tabular}

* Teste Kruskal-Wallis de amostras independentes; TTC: Termo de tendência central; *CH- Concentrado de Hemácias, CP- Concentrado de Plaquetas, CPlasma- Concentrado de Plasma, ST- Sangue Total. Fonte: Autores.

Quando considerados os descartes das bolsas no setor de distribuição dos hemocomponentes foi notado que o $\mathrm{CH}$, CP, CPlasma, apresentaram termos de tendências central semelhantes sobre os expurgos das bolsas (Quadro 3).

Quadro 3: Descartes dos concentrados devido intercorrência na distribuição durante o período de setembro de 2017 a agosto de 2018 no Centro de Hemoterapia de Sergipe.

\begin{tabular}{|l|c|c|c|c|}
\hline \multicolumn{5}{|c|}{ TERMOS DE TENDÊNCIA CENTRAL } \\
TIPOS & MEDIANA & MÍNIMO & MÁXIMO & \multirow{2}{*}{$\mathbf{0 . 1 4 2}$} \\
\hline CH & 1 & 0 & 2 & \\
\hline CP & 1 & 0 & 2 \\
\hline CPlasma & 1 & 0 & 2 \\
\hline ST & - & - & - \\
\hline
\end{tabular}

* Teste Kruskal-Wallis de amostras independentes; TTC: Termo de tendência central; *CH- Concentrado de Hemácias, CP- Concentrado de Plaquetas, CPlasma- Concentrado de Plasma, ST- Sangue Total. Fonte: Autores.

Nos procedimentos operacionais do setor de dispensação de bolsas de hemocomponentes foram observadas tendências central semelhantes no CH, CP, CPlasma (Quadro 4).

Quadro 4: Descartes dos concentrados devido intercorrência no estoque durante o período de setembro de 2017 a agosto de 2018 no Centro de Hemoterapia de Sergipe.

\begin{tabular}{|l|l|c|c|c|}
\hline \multicolumn{5}{|c|}{ TERMOS DE TENDÊNCIA CENTRAL } \\
\multirow{2}{*}{ TIPOS } & MEDIANA & MÍNIMO & MÁXIMO & \multirow{2}{*}{$\mathbf{0 . 3 4 6}$} \\
\hline CH & CH & 1 & 0 \\
\hline CP & CP & 1 & 0 \\
\hline CPlasma & CPlasma & 1 & 0 \\
\hline ST & ST & - & - \\
\hline
\end{tabular}

* Teste Kruskal-Wallis de amostras independentes; TTC: Termo de tendência central; *CH- Concentrado de Hemácias, CP- Concentrado de Plaquetas, CPlasma- Concentrado de Plasma, ST- Sangue Total. 
Em relação a centrifugação e o fracionamento os maiores índices de intercorrências ocorreu com CPlasma, seguido de CP, (Quadro 5).

Quadro 5: Descartes dos concentrados devido intercorrência no fracionamento durante o período de setembro de 2017 a agosto de 2018 no Centro de Hemoterapia de Sergipe.

\begin{tabular}{|l|c|c|c|c|}
\hline \multicolumn{5}{|c|}{ TERMOS DE TENDÊNCIA CENTRAL } \\
\hline TIPOS & MEDIANA & MÍNIMO & MÁXIMO & \multirow{2}{*}{$\mathbf{0 . 0 0 4}$} \\
\hline CH & 2 & 0 & 57 & \\
\hline CP & 15 & 0 & 102 & \\
\hline CPlasma & 35.5 & 0 & 260 \\
\hline ST & 5 & 1 & 14 & \\
\hline
\end{tabular}

* Teste Kruskal-Wallis de amostras independentes *CH- Concentrado de Hemácias, CP- Concentrado de Plaquetas, CPlasma- Concentrado de Plasma, ST- Sangue Total.

Fonte: Autores.

Baseado no quadro supracitado, percebe-se que a distribuição dos concentrados apresentou diferença estatística $(\mathrm{p}<0,05)$, sendo que os concentrados de plaquetas, apresentou um maior índice de descarte, enquanto o concentrado de plasma apresentou maior valor de descarte. Quando aplicado o post-hoc test para comparação entre os pares, notou-se que a diferença estatística era prevalente entre o sangue total e o concentrado de plasma $(\mathrm{p}=0,017)$.

Figura 1: Post-hoc teste para comparação entre os concentrados de hemácias, plaquetas, plasma e sangue total durante o período de setembro de 2017 a agosto de 2018 no Centro de Hemoterapia de Sergipe.

Comparação Entre Pares de Tipo

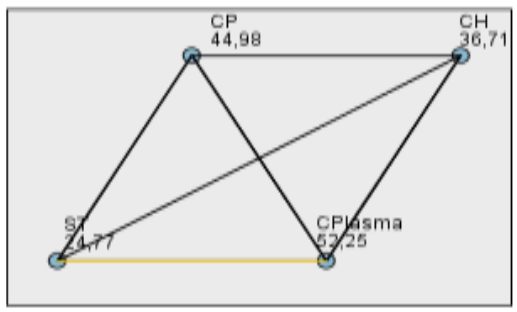

Cada nó mostra a posição média de amostra de Tipo.

\begin{tabular}{|lrrrrrr|}
\hline Amostra1-Amostra2 & $\begin{array}{c}\text { Estatistica de } \\
\text { Teste }\end{array}$ & $\begin{array}{c}\text { Std. } \\
\text { Erro }\end{array}$ & $\begin{array}{c}\text { Erro Estatistica de } \\
\text { Teste }\end{array}$ & Sig. & Sig. Aj. \\
\hline ST-CH & 11,939 & 7,975 & 1,497 &, 134 &, 806 \\
\hline ST-CP & 20,203 & 8,526 & 2,370 &, 018 &, 107 \\
\hline ST-CPlasma & 27,477 & 9,229 & 2,977 &, 003 &, 017 \\
\hline CH_CP & $-8,264$ & 6,394 & $-1,292$ &, 196 & 1,000 \\
\hline CH-CPlasma & $-15,538$ & 7,306 & $-2,127$ &, 033 &, 201 \\
\hline CP-CPlasma & $-7,274$ & 7,904 &,- 920 &, 357 & 1,000 \\
\hline
\end{tabular}

Cada linha testa a hipótese nula de que as distribuiçōes da Amostra 1 e da Amostra 2 são as Săo exibidas significåncias assintóticas (teste de 2 lados). O nivel de significåncia $\theta, 05$.

Fonte: Autores. 


\section{Discussão}

Os descartes de concentrados de hemácias, plaquetas, plasma e sangue total, pode ser atribuído a falta de verificação dos procedimentos operacionais deste a coleta, processamento e estocagem (Collins, et al., 2015). Pesquisas demonstram que o monitoramento do sistema de qualidade na dispensação e reintegração dos hemocomponentes acarreta na minimização do desperdício das unidades de sangue (Chung, et al., 2016).

O índice percentual de produção, transfusão, descarte e modificação de hemocomponentes durante o ano de 2018 foi de 33,26\% (2.640.486) (Hemoprod, 2018)

A distribuição dos concentrados apresentou diferença estatística $(\mathrm{p}<0,05)$, sendo que os concentrados de plaquetas, apresentou um maior índice de descarte, enquanto o concentrado de plasma apresentou maior valor de descarte. Quando aplicado o post-hoc test para comparação entre os pares, notou-se que a diferença estatística era prevalente entre o sangue total e o concentrado de plasma $(\mathrm{p}=0,017)$.

Com base na análise dos dados relacionados ao descarte de hemocomponentes observou que teste coombs direto, sorologia reagente, bolsas fora da norma RDC 24/02, hemoglobina S positiva, agregação plaquetária e falta de local para armazenamento apresentaram significância estatística $(\mathrm{p}<0,05)$

Os Serviços de Hemoterapia e Hematologia (HEMORREDE) de Brasília, através de relatórios mensais no período de 2013 a 2015, distribuição de hemocomponentes nas agências transfusionais da Hemorrede do Distrito Federal, a partir do índice de descarte/expurgo, por vencimento, de bolsas de concentrado de hemácias demonstrou que as bolsas de concentrados de hemácias foram as que tiveram maior índice de descarte por expiração do prazo de validade (Reis, et al., 2017).

No território brasileiro, análises demonstraram heterogeneidade na incidência de expurgo de bolsas contendo concentrado de hemácias devido a validade expirada, em Hemocentro do estado de Porto Alegre apresentou uma incidência de descarte entre 4,35\% a 61,46\%, (Fonte, 2004). Enquanto no estado no Paraná durante o ano de 2010 a 2012 os expurgos das bolsas variaram entre $1,76 \%$ a 1,85\% denotados índices menores encontrado no presente estudo. (Moura, 2013)

O descarte de bolsas por dúvidas clínicas na triagem e volume pode ser atribuído a vários fatores como características dos doadores, experiência dos profissionais responsáveis pela triagem, descuido dos triadores ou até mesmo a necessidade de agilizar o atendimento, o que pode acarretar em falta de tempo para avaliação cuidadosa da conduta na triagem (Brasil, 2018).

O rompimento de bolsas de sangue é uma das causas com maior incidência, sua origem pode ser devido a razões externas ou internas ao hemocentro. Externas quando ocorrem em função de desvios como produção de bolsas com soldagem fraca ou matérias com resistência física inferior o que compromete sua resistência física, manuseio das bolsas, estocagem, transporte e vários outros fatores (Cândido, 2019).

Com relação aos desvios internos (no hemocentro), ocorrem principalmente devido ao manuseio, intercorrência no processamento e armazenamento, transporte inadequado das bolsas antes, durante ou após seu acondicionamento. (Covo, 2018)

O prazo de validade vencido, é mais uma causa de descarte com incidência significativa, que pode ser a causa primaria de descarte a depender do hemocomponente (Ferreira, 2013). As ocorrências podem ser acidentais (erro humano) ou por intercorrência nos estoques devido à ausência de procedimento adequado de estocagem e anteposição dos produtos com prazo de expiração maior, ocasionando perda dos que possuem prazo de validade menor. (Hosn, 2009)

Numerosos casos de descartes sucedidos de forma desnecessária em Hemocentros poderiam ser contidos com programas simples e constantes de qualificação dos profissionais envolvidos, entre outras ações, concernente a um Sistema de Gestão de Qualidade. Com a realização das medidas citadas, ocorreria a diminuição das necessidades de produção, assim como custos sociais e financeiros consequentes da perda dos produtos (Anvisa, 2017). 


\section{Considerações Finais}

Mediante o estudo realizado em um centro de Hemoterapia de uma região do nordeste do Brasil no período de setembro de 2107 a agosto de 2018 tornou-se possível analisar a gestão do descarte de hemocomponentes através do índice de inaptidão das bolsas de sangue.

Por meio das informações coletadas nos registros de descarte de sangue total e hemocomponentes do Hemocentro, os dados apresentaram uma média de coleta de 25.000 bolsas de sangue, destas, 5.828 foram descartadas em recorrência de variáveis como intercorrência no fracionamento, intercorrência na distribuição, expiração do prazo de validade, acondicionamento inadequado, sorologia reagente, coombs indireto e intercorrência no estoque.

Os resultados do presente estudo propõem um plano de ação, envolvendo a reavaliação dos protocolos operacionais, com avaliações periódicas das demandas de descarte, visando readequar a oferta e reduzir ou até extinguir o descarte de bolsas de hemocomponentes, o que direcionaria a uma redução de custos financeiros e perdas sociais.

O corrente estudo não exaure a temática abordada, todavia, os dados fornecidos pelo Hemocentro devem ser aprofundados para que ocorra a identificação e possível correção na unidade. As recomendações aqui citadas podem ser adequadas e implantadas em outros hemocentros e unidades de saúde.

\section{Referências}

Agência Nacional De Vigilância Sanitária (ANVISA). (2017). $4^{o}$ Boletim Anual de Produção Hemoterápica. Brasília, DF.

Agência Nacional De Vigilância Sanitária (ANVISA). (2018). $7^{\circ}$ Boletim de Produção Hemoterápica - Hemoprod. Brasília, DF.

Brasil. Ministério da Saúde. (2016). Portaria $n^{\circ}$ 158, de 4 de fevereiro de 2016. Redefine o regulamento técnico de procedimentos hemoterápicos. PRT MS/GM 158/2016, Art. 5 XI. Diário Oficial da República Federativa do Brasil, Brasília, DF: Gabinete do Ministro da Saúde.

Brasil. Ministério da Saúde. (2018). Gestão de Hemocentros: relatos de práticas desenvolvidas no Brasil. Brasília - DF.

Cândido, A. L. P. \& Danta, D. C. (2019). Incidência de transfusões de plaquetas não isogrupo em pacientes atendidos pelo hemocentro - ATHC de Ribeirão Preto. Monografia apresentada ao Programa de Aprimoramento Profissional/CRH/SES-SP, elaborada no Hospital das Clínicas da Faculdade de Medicina de Ribeirão Preto da Universidade de São Paulo - USP/ Departamento Hemocentro.

Chung, K. W., Basavaraju, S. V., M, U. Y., Santen, K. L., Haass, K. A., Henry, R., Berger, J. \& Kuehnert, M. J. (2016) Declining blood collection and utilization in the United States. Transfusion. 56, 2184-2192.

Collins, R. A., Wisniewski, M. K., Waters, J. H., Triulzi, D. J. \& Yazer, M. H. (2015). Effectiveness of Multiple Initiatives to Reduce Blood Component Wastage. Am J Clin Pathol. 14(3), 329-335.

Covo, M. Z. (2018). Matriz de recomendações para melhoria de desempenho do ciclo do sangue no hemocentro coordenador do estado do Paraná. Dissertação apresentada ao Curso de Mestrado Profissional do Programa de Pós-Graduação em Enfermagem.

Covo, M. Z., Cruz, E. D. A., Maurício, A. B., Batista, J. \& Souza, L. A. L. (2019). Custo financeiro dos descartes de sangue total e hemocomponentes em um hemocentro coordenador brasileiro. Rev Gaúcha Enferm. 40, e20190033.

Dias, P., Lissa, N., Skare, T., Fávero, K., Almeida, P. \& Nishiara, R. (2019). Pre-donation deferral of blood donors in a Brazilian blood bank: a 10-year experience. Transfusion Medicine. 29(6), 448-453.

Ferreira, A. M. (2013). Produção, armazenamento, distribuição e transporte de Hemocomponentes. In: BRASIL. Ministério da Saúde. Técnico em hemoterapia: livro texto. Brasília, DF.

Fonte, C. A. G. (2004). Como otimizar a utilização do sangue no estado do Rio Grande do Sul. Trabalho de conclusão de curso - Programa de Desenvolvimento da Gestão em Saúde - PDG. Universidade Federal do Rio Grande do Sul.

Hosn, C. U. R. C. A. (2009). Análise do descarte de hemocomponentes no Hemocentro Regional de Araguaína - TO. Goiânia- GO.

Moura, E. (2013). Identificação dos fatores de descarte de bolsas na produção de hemocomponentes no hemocentro coordenador da rede HEMEPAR.

Organización Panamericana de la Salud (US). (2017). Suministro de sangre para transfusiones en los países de Latinoamérica y del Caribe 2014 y 2015. Washington, DC.

Paludetto, N. O. (2015). Implantação de gestão da qualidade no serviço de hemoterapia em um hospital público do Estado de São Paulo. Universidade Estadual Paulista, Campus de Botucatu. Mestrado em Pesquisa e Desenvolvimento. 
Research, Society and Development, v. 10, n. 10, e540101019344, 2021

(CC BY 4.0) | ISSN 2525-3409 | DOI: http://dx.doi.org/10.33448/rsd-v10i10.19344

Reis, D. J. C., Aleluia, I. R. S., Martins, M. M. F. \& Pinto, J. E. P. (2017). Análise da distribuição de hemocomponentes na hemorrede do Distrito Federal. Arq. Cienc. Saúde UNIPAR, Umuarama. 21(2), 93-98.

Secretária de Estado da Saúde. (2020). Plano Estadual de Saúde 2020-2023. Governo do Estado do Rio Grande do Sul. Porto Alegre 2020.

Ventura, C. (2019). Dezesseis a cada mil brasileiros doam sangue. Ministério da Saúde do Brasil - Agência Saúde.

World Health Organization (WHO). (2017). Blood safety and availability. 